\title{
As lacunas do cuidado de enfermagem às pessoas com incontinência urinária
}

\author{
Gaps in nursing care for people with urinary incontinence
}

\section{Las lagunas del cuidado de enfermería para las personas con incontinencia urinaria}

Sarah Cristiane dos Reis', Thiago Suzano Oliveira', Eliza Maria Rezende Dázio ${ }^{1,2}$, Roberta Seron Sanches ${ }^{1,2}$, Jenika Ferreira Dias ${ }^{1,3}$, Silvana Maria Coelho Leite Fava ${ }^{1,2}$

ORCID IDs

Reis SC (D) https://orcid.org/0000-0002-2801-9032

TS Oliveira (iD https://orcid.org/0000-0002-0782-8957

Dázio EMR (iD https://orcid.org/0000-0001-9216-6283

Sanches RS (iD https://orcid.org/0000-0001-7557-5560

Dias JF (D) https://orcid.org/0000-0002-2743-0751

Fava SMCL (iD https://orcid.org/0000-0003-3186-9596

\section{COMO CITAR}

Reis SC, Oliveira TS, Dázio EMR, Sanches RS, Dias JF, Fava SMCL. As lacunas do cuidado de enfermagem às pessoas com incontinência urinária. ESTIMA, Braz. J. Enterostomal Ther., 16: e3618. https://doi. org/10.30886/estima.v16.621 PT

\section{RESUMO}

Objetivos: Levantar na literatura o papel do enfermeiro no cuidado às pessoas com incontinência urinária (IU). Métodos: Revisão integrativa da literatura a partir das publicações no período de 2013 nas bases e bancos de dados: BDENF, LILACS, Web of Science, PubMed e SciELO, nos idiomas português, inglês e espanhol. A amostra foi composta por 11 publicações, sendo a maioria em inglês. Resultados: Os dados foram categorizados nas três dimensões do cuidado: educacional (três artigos), assistencial (oito artigos) e gerencial (um artigo, o qual também foi categorizado como assistencial). Educacional: embora os enfermeiros tenham apresentado conhecimento superficial e incompleto sobre a incontinência, foram utilizadas estratégias lúdicas para o ensino. Assistencial: foram recomendados o uso de fraldas e almofadas superabsorventes, lenços umedecidos e impregnados com dimeticona a 3\%, spray para formação de película de polímero para dermatite associada à incontinência, exercício da musculatura pélvica, massagem uretral, treinamento da bexiga e modificação dos hábitos alimentares. Gerencial: avaliou-se a qualidade do atendimento de enfermagem aos idosos com IU por meio de protocolos, normas e Procedimentos Operacionais Padrão (POPs). Conclusão: Há escassez de estudos na temática e evidenciou-se que o enfermeiro assume papel assistencial na incontinência urinária, embora seu conhecimento seja incipiente.

DESCRITORES: Enfermagem; Incontinência urinária; Cuidados de enfermagem; Estomia; Estomaterapia.

\footnotetext{
${ }^{1}$ Universidade Federal de Alfenas - Escola de Enfermagem - Curso de Graduação em Enfermagem - Alfenas/MG - Brasil.

${ }^{2}$ Universidade Federal de Alfenas - Escola de Enfermagem - Programa de Pós-Graduação em Enfermagem - Alfenas/MG - Brasil. ${ }^{3}$ Pontifícia Universidade Católica de Campinas - Programa de Pós-Graduação em Urgência e Emergência - Campinas/SP - Brasil. Autor correspondente: Silvana Maria Coelho Leite Fava | Rua Gabriel Monteiro da Silva, 700 - Centro | CEP: $37130-000$ - Alfenas/MG - Brasil | E-mail: silvanalf2005@yahoo.com.br Recebido: Jul 122018 | Aceito: Jan 132019
} 


\begin{abstract}
Objective: To survey in the literature the role of the nurse in the care of people with urinary incontinence (UI). Methods: Integrative literature review from the publications within the period of 2013 in the databases: BDENF, LILACS, Web of Science, PubMed and SciELO, in the Portuguese, English and Spanish languages. The sample consisted of 11 publications, most of them in English. Results: The data were categorized into three dimensions of care: educational (three articles), care (eight articles) and management (an article, which was also categorized as care). Educational: although nurses presented superficial and incomplete knowledge about incontinence, playful strategies were used for teaching. Assistance: the use of superabsorbent diapers and pads, 3\% dimethicone impregnated wipes, spray for the formation of polymer film for incontinence-associated dermatitis, pelvic musculature, urethral massage, bladder training, and eating habits modification were recommended. Gerencial: the quality of nursing care for the elderly with UI was evaluated through protocols, standards and Standard Operational Procedures (SOPs). Conclusion: There is a shortage of studies on the subject and it was evidenced that the nurse assumes an assistance role in urinary incontinence, although its knowledge is incipient.
\end{abstract}

DESCRIPTORS: Nursing; Urinary incontinence; Nursing care; Ostomy; Stomatherapy.

\title{
RESUMEN
}

Objetivos: Levantar en la literatura el papel del enfermero en el cuidado a las personas con incontinencia urinaria (IU). Métodos: Revisión integral de la literatura a partir de las publicaciones en el período del 2013 en las bases de datos: BDENF, LILACS, Web of Science, PubMed y SciELO, en los idiomas portugués, inglés y español. La muestra estuvo compuesta por 11 publicaciones, siendo la mayoría en inglés. Resultados: Los datos fueron categorizados en las tres dimensiones del cuidado: educativo (tres artículos), asistencial (ocho artículos) y gerencial (un artículo, el cual también fue categorizado como asistencial). Educativo: a pesar de que los enfermeros hayan presentado conocimiento superficial e incompleto sobre la incontinencia, fueron utilizadas estrategias lúdicas para la enseñanza. Asistencial: fue recomendado el uso de pañales y almohadillas super-absorbentes, toallas húmedas e impregnadas con dimeticona al 3\%, spray para formación de película de polímero para dermatitis asociada a la incontinencia, ejercicio de la musculatura pélvica, masería uretral, entrenamiento de la vejiga y modificación de los hábitos alimentarios. Gerencial: se evaluó la calidad del servicio de enfermería a los ancianos con IU por medio de protocolos, normas y Procedimientos Operativos Estándar (POPS). Conclusión: Hay escasez de estudios en la temática y se evidenció que el enfermero asume el papel asistencial en la incontinencia urinaria, a pesar de que su conocimiento sea incipiente.

DESCRIPTORES: Enfermería; Incontinencia urinaria; Cuidados de enfermería; Ostomía; Estomaterapia.

\section{INTRODUÇÃO}

A incontinência urinária (IU) é caracterizada como qualquer perda involuntária de urina e é classificada em três tipos: IU de esforço (IUE), caracterizada pela queixa de perda urinária por qualquer esforço, espirros ou tosse; IU de urgência (IUU), a qual ocorre devido a vazamento precedido por urgência; e IU mista (IUM), originada pela associação dos dois tipos de $\mathrm{IU}^{1}$.

A IU constitui importante problema de saúde pública, tendo em vista suas altas taxas na população e os impactos negativos que podem ocasionar na vida de uma pessoa, comprometendo sua qualidade de vida, a interação social, as atividades de vida diária e a vida sexual².

É importante considerar que os índices de prevalência e de incidência de pessoas com IU serão diferentes, a depender do contexto geográfico e social e das características populacionais, tais como idade, sexo, condições físicas e mentais. Assim, observa-se que a prevalência de pessoas com IU é de $27,6 \%$ no gênero feminino e $10,5 \%$ no gênero masculino ${ }^{3}$.

Com relação à causalidade da IU, evidencia-se que pode estar associada a inúmeros fatores, como: idade avançada; histórico obstétrico e paridade; cirurgias ginecológicas; menopausa; hipertensão arterial sistêmica; terapia com diuréticos e outros medicamentos; diabetes melito; obesidade; tabagismo; álcool; ingestão de líquidos cafeinados; sedentarismo; hiperplasia de próstata; e sedentarismo.

A enfermagem assume papel fundamental. A atuação do enfermeiro se desenvolve a partir do acolhimento, do estabelecimento do vínculo, propondo ações que minimizem os danos, realizando ações de promoção e prevenção de agravos e elaboração de protocolos. 


\section{OBJETIVOS}

Levantar, analisar e categorizar a produção científica acerca do papel do enfermeiro no cuidado às pessoas com IU e apresentar os resultados encontrados.

\section{MÉTODOS}

Trata-se de revisão integrativa (RI) da literatura na qual foram seguidas as seis seguintes etapas: identificação do tema e seleção da hipótese; estabelecimento de critérios para inclusão e exclusão de estudos; definição das informações a serem extraídas dos estudos selecionados e categorização dos estudos; avaliação dos estudos incluídos na RI; interpretação dos resultados; e apresentação da revisão/síntese do conhecimento ${ }^{4}$.

Inicialmente, elaborou-se a seguinte questão norteadora: qual o papel do enfermeiro no cuidado às pessoas com IU? A busca dos estudos foi realizada online nas bases e bancos de dados. Foram definidos, como critérios de inclusão, artigos nos idiomas português, inglês e espanhol e publicados no período compreendido entre 2013 a 2017. Justifica-se esse recorte temporal, tendo em vista a necessidade de se levantar a produção atual da enfermagem na temática e as lacunas do conhecimento para subsidiar novos estudos ${ }^{4}$.

As buscas foram realizadas nas bases e bancos de dados Bases de Dados em Enfermagem (BDENF), Literatura Latino-Americana e do Caribe em Ciências da Saúde (LILACS), Web of Science, National Library of Medicine National - Institute of Health (PubMed), que engloba a MEDLINE, e Scientific Electronic Library Online (SciELO), por meio dos seguintes descritores: Enfermagem, Nursing, Enferméria, Cuidados de Enfermagem, Nursing Care, Atencion Enferméria, Estomia, Stomy, Estomia, Incontinência Urinária e Incontinence Urinary utilizando o operador booleano "AND" entre os descritores ${ }^{4}$.

Para obtenção dos dados, utilizou-se um instrumento validado que contempla os seguintes dados: ano de publicação; idioma; autoria; objetivo do estudo; método; resultados; conclusões; níveis de evidência; e lacunas do conhecimento 5 . Para avaliar os níveis de evidência, adotaram-se critérios preestabelecidos, os quais preconizam uma hierarquia de evidências de acordo com o tipo de estudo ${ }^{6}$.

Posteriormente, realizou-se a análise das informações por meio da leitura dos artigos selecionados. Os dados foram, então, organizados e categorizados a partir de temas recorrentes, de acordo com as dimensões do cuidado, o que permitiu o alcance do objetivo do presente estudo ${ }^{7}$.

\section{RESULTADOS}

Após o levantamento dos dados, realizou-se o processo de seleção dos estudos que se adequaram aos critérios de inclusão, conforme mostra a Fig. 1.

Para avaliação dos estudos, os dados foram categorizados como demonstra o Tabela 1. Constatou-se que a maioria dos estudos $36,37 \%(n=4)$ foi publicada em $2013,54,54 \%(\mathrm{n}=6)$ no idioma inglês e $27,27 \%$ $(\mathrm{n}=3)$ no Brasil.

Com relação ao delineamento do estudo, observou-se que $27,27 \%(\mathrm{n}=2)$ adotaram abordagem qualitativa. Quanto aos níveis de evidências, houve predomínio de 54,54\% ( $\mathrm{n}=6$ ) classificados com o nível de evidência VI, de acordo com o Tabela 2.

Com relação aos resultados, os dados foram agrupados em categorias de análise, devido à similitude da temática, e relacionados às três dimensões do cuidado: educacional, assistencial e gerencial.

\section{Primeira dimensão do cuidado: educacional}

Essa categoria contempla as temáticas relacionadas à educação com foco na prevenção, no tratamento e na autogestão, com ênfase nas tecnologias, nos dispositivos e na aplicação de técnicas e intervenções. Assim, incluíram-se três artigos, sendo que dois abordaram questões acerca da prevenção e do tratamento, enquanto um discutiu acerca da autogestão, como podemos evidenciar a seguir.

Um estudo desenvolvido com enfermeiros evidenciou que todos apresentaram conhecimento superficial acerca da IU e, ainda, mencionaram que faziam ações 


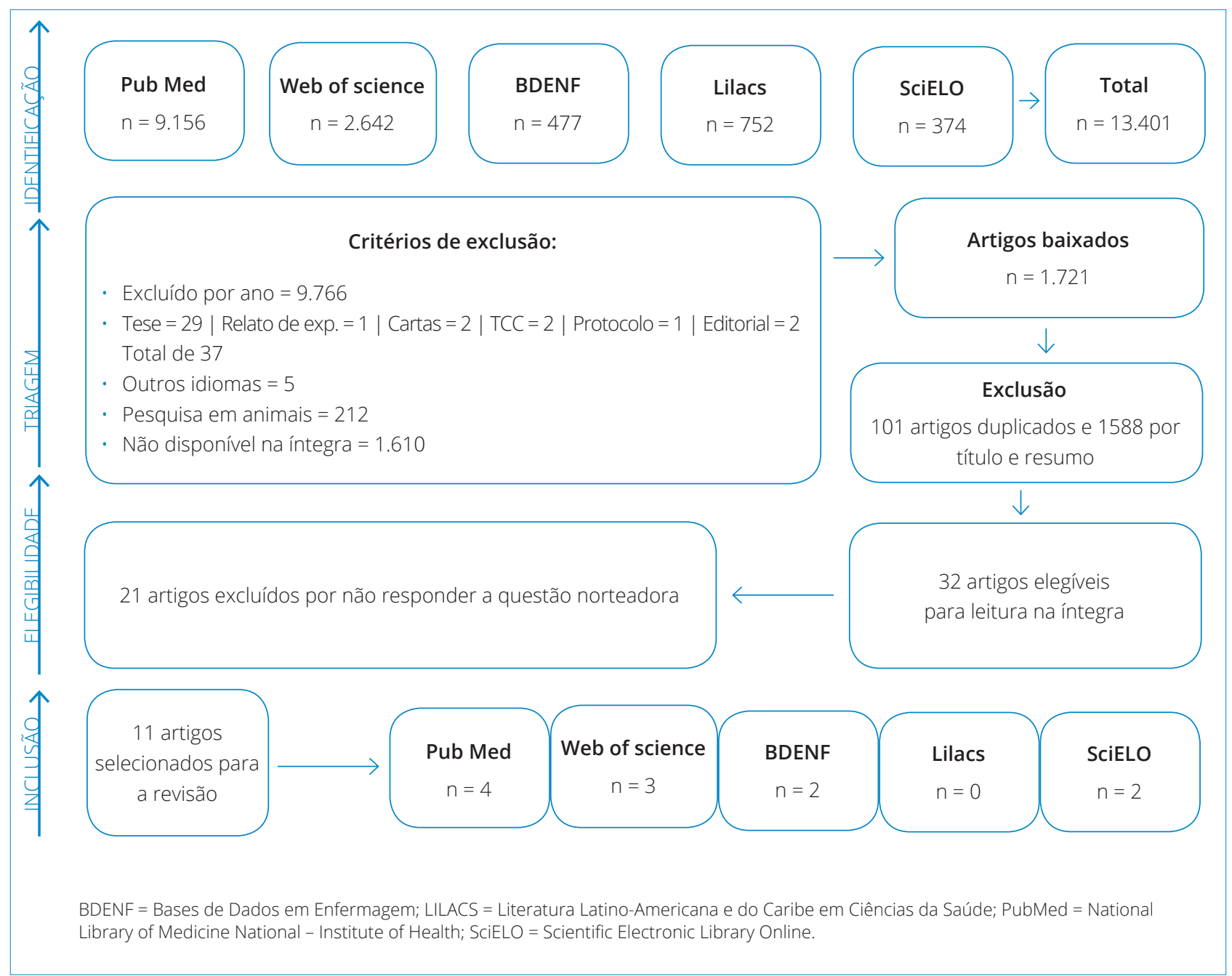

Figura 1. Fluxograma processo de seleção dos artigos. Adaptação do flow diagrama do processo de seleção de artigos da revisão integrativa, de acordo com o Preferred Reporting Items for Systematic Reviews and Meta-Analyses (PRISMA).

de prevenção, mas, na realidade, a atuação limitava-se ao tratamento e à reabilitação de pessoas que buscavam o atendimento na Estratégia de Saúde da Família (ESF). Verificou-se, ainda, que apenas um enfermeiro preocupou-se em incentivar o autocuidado no manejo da $\mathrm{IU}^{8}$.

Para identificar o conhecimento e as práticas assistenciais acerca da IU em mulheres idosas e desenvolver a proposta de cuidado para promoção da saúde no âmbito da atenção primária, realizou-se entrevista com 14 fisioterapeutas e 10 enfermeiros, em um distrito sanitário de saúde de uma capital do sul do Brasil. Ao final desse estudo, os autores destacaram que o conhecimento dos profissionais sobre as medidas de prevenção e de tratamento da IU é de suma importância para o acolhimento e o estabelecimento de vínculo, pois pode facilitar e sensibilizar as pessoas a manifestarem suas queixas e a buscarem pelo tratamento 9 .
Em um programa educacional de autogestão da IU, utilizaram-se atividades que envolviam a ludicidade, as quais eram executadas por meio de músicas, cadernos de desenho, adesivos coloridos e discussões reflexivas. Essas atividades tinham o propósito de modificar os fatores de risco da IU, como: ingestão inadequada de dieta e líquidos; irregularidade intestinal; excesso de peso; tabagismo; inatividade física; e musculatura do assoalho pélvico enfraquecido. Os exercícios para fortalecer a musculatura do assoalho pélvico eram realizados por meio dos exercícios de Kegel, bolas de ioga e bolas de praia. Os resultados evidenciaram que os participantes ficaram muito satisfeitos com a eficácia das atividades desenvolvidas no programa, uma vez que, além de terem promovido a aquisição de conhecimentos, minimizaram a gravidade dos sintomas ocasionados pela $\mathrm{IU}^{10}$. 
Tabela 1. Caracterização dos estudos sobre incontinência urinária segundo título, periódico, ano de publicação, idioma e país, 2013-2017, Alfenas/MG, Brasil.

\begin{tabular}{|c|c|c|c|c|}
\hline Título & $\begin{array}{c}\text { Periódico } \\
\text { (Tipo de publicação) }\end{array}$ & $\begin{array}{c}\text { Ano de } \\
\text { publicação }\end{array}$ & Idioma & País \\
\hline $\begin{array}{l}\text { Assistência de enfermagem a idosos com } \\
\text { incontinência urinária }\end{array}$ & $\begin{array}{l}\text { Revista de Enfermagem UFPE } \\
\text { (Artigo original) }\end{array}$ & 2017 & Português/inglês & Brasil \\
\hline $\begin{array}{l}\text { Incontinência urinária em idosas: práticas } \\
\text { assistenciais e proposta de cuidado âmbito da } \\
\text { atenção primária de saúde }\end{array}$ & $\begin{array}{l}\text { Texto \& Contexto Enfermagem } \\
\text { (Artigo original ) }\end{array}$ & 2017 & Português & Brasil \\
\hline $\begin{array}{l}\text { Tratamento da dermatite associada à } \\
\text { incontinência em idosos institucionalizados: } \\
\text { revisão integrativa }\end{array}$ & $\begin{array}{l}\text { Revista da Rede de } \\
\text { Enfermagem do Nordeste } \\
\text { (Artigo de revisão) }\end{array}$ & 2013 & Português & Brasil \\
\hline $\begin{array}{l}\text { Interventions for preventing and treating } \\
\text { incontinence-associated } \\
\text { dermatitis in adults }\end{array}$ & $\begin{array}{l}\text { Cochrane Database of } \\
\text { Systematic Reviews } \\
\text { (Artigo de revisão) }\end{array}$ & 2016 & Inglês/espanhol & Bélgica \\
\hline $\begin{array}{l}\text { Medidas preventivas para evitar complicaciones } \\
\text { de salud derivadas del uso de sondajes } \\
\text { vesicales en pacientes lesionados medulares }\end{array}$ & $\begin{array}{c}\text { Enfermería Global } \\
\text { (Artigo de revisão integrativa) }\end{array}$ & 2013 & Espanhol & Espanha \\
\hline $\begin{array}{l}\text { Vaginal pessaries for pelvic organ prolapse } \\
\text { and urinary incontinence: a multiprofessional } \\
\text { survey of practice }\end{array}$ & $\begin{array}{l}\text { Jornal Internacional de } \\
\text { Uroginecologia } \\
\text { (Artigo original) }\end{array}$ & 2013 & Inglês & Reino Unido \\
\hline $\begin{array}{l}\text { Incontinence care in nursing homes: a cross- } \\
\text { sectional study }\end{array}$ & $\begin{array}{l}\text { Journal of Advanced Nursing } \\
\text { (Artigo original) }\end{array}$ & 2015 & Inglês & Áustria \\
\hline $\begin{array}{l}\text { Twelve-year follow-up of conservative } \\
\text { management of postnatal urinary and faecal } \\
\text { incontinence and prolapse outcomes: } \\
\text { randomised controlled trial }\end{array}$ & $\begin{array}{l}\text { International Journal of } \\
\text { Obstetrics and Gynaecology } \\
\text { (Artigo original) }\end{array}$ & 2013 & Inglês & $\begin{array}{l}\text { Reino Unido/ } \\
\text { Nova Zelândia }\end{array}$ \\
\hline $\begin{array}{l}\text { Treatment by a nurse practitioner in primary } \\
\text { care improves the severity and impact } \\
\text { of urinary incontinence in women. An } \\
\text { observational study }\end{array}$ & $\begin{array}{l}\text { Bio Med Central Urology } \\
\text { (Artigo original) }\end{array}$ & 2015 & Inglês & Holanda \\
\hline $\begin{array}{l}\text { The effect of a urinary incontinence self } \\
\text { management program for older women in } \\
\text { South Korea: A pilot study }\end{array}$ & $\begin{array}{c}\text { International Journal of Nursing } \\
\text { Sciences } \\
\text { (Artigo original) }\end{array}$ & 2015 & Inglês & Coreia do Sul \\
\hline $\begin{array}{l}\text { Evaluation of the effectiveness of nurse-led } \\
\text { primary care patients with lower urinary tract } \\
\text { symptoms }\end{array}$ & $\begin{array}{l}\text { Plos One } \\
\text { (Artigo original) }\end{array}$ & 2015 & Inglês & China \\
\hline
\end{tabular}

Adaptado de Mendes et al. (2008).

\section{Segunda dimensão do cuidado: assistencial}

$\mathrm{Na}$ categoria assistencial, selecionaram-se sete artigos, sendo que três contemplavam a aplicação de técnicas/ intervenções e quatro tinham foco nas tecnologias, dispositivos e outros produtos, como mencionados a seguir.

Ensaio clínico randomizado foi realizado no Reino Unido e na Nova Zelândia, o qual teve o objetivo de determinar os efeitos de uma intervenção conservadora ativa (treinamento do músculo do assoalho pélvico e da bexiga para a IU pós-natal), por um período de 12 anos.
A intervenção foi realizada por enfermeiro capacitado que orientou e conduziu os exercícios da musculatura pélvica e da bexiga em três ocasiões: aos 5, 7 e 9 meses após o parto, para que as mulheres tivessem condições de realizá-los no decorrer de 1 e 6 anos. Após 12 anos, as mulheres foram contatadas e constatou-se a prevalência de IU em quatro quintos das participantes. Ao final, os autores concluíram que, em longo prazo, a intervenção não teve nenhum efeito na redução da IU e que o resultado poderia ter sido melhor se fossem realizados encontros para treinamento contínuo desses exercícios ${ }^{11}$. 
Tabela 2. Caracterização dos artigos sobre incontinência urinária (IU) segundo os autores, objetivo, tipo de estudo e níveis de evidência, 2013-2017, Alfenas/MG, Brasil.

\begin{tabular}{|c|c|c|c|}
\hline Autor & Objetivo & Tipo de estudo & $\begin{array}{l}\text { Nível de } \\
\text { evidências }\end{array}$ \\
\hline Bittencourt et al. & Descrever a assistência de enfermagem prestada ao idoso com IU & $\begin{array}{l}\text { Descritivo/ } \\
\text { qualitativo }\end{array}$ & $N-6$ \\
\hline Tomasi et al. & $\begin{array}{l}\text { Identificar o conhecimento e as práticas assistenciais sobre a IU em } \\
\text { mulheres idosas e desenvolver proposta de cuidado a essas mulheres } \\
\text { para a promoção da saúde no âmbito da atenção primária de saúde }\end{array}$ & $\begin{array}{l}\text { Descritivo- } \\
\text { qualitativo }\end{array}$ & N-6 \\
\hline Rosa et al. & $\begin{array}{l}\text { Identificar opções de intervenção terapêutica dos últimos } 5 \text { anos } \\
\text { para tratamento da dermatite associada à incontinência em idosos } \\
\text { institucionalizados }\end{array}$ & $\begin{array}{l}\text { Revisão } \\
\text { integrativa }\end{array}$ & N-6 \\
\hline Beeckman et al. & $\begin{array}{l}\text { Avaliar a eficácia de vários produtos e procedimentos para prevenir e } \\
\text { tratar a dermatite associada à incontinência em adultos }\end{array}$ & $\begin{array}{l}\text { Descritivo/ } \\
\text { qualitativo }\end{array}$ & N-6 \\
\hline Torres \& Agustias & $\begin{array}{l}\text { Determinar se certos tipos de sondas e técnicas são melhores para a } \\
\text { prevenção de complicações na IU em longo prazo }\end{array}$ & $\begin{array}{l}\text { Revisão } \\
\text { integrativa }\end{array}$ & N-6 \\
\hline Bugee et al. & $\begin{array}{c}\text { Investigar perspectivas multidisciplinares sobre pessários vaginais na } \\
\text { prática clínica para entender a organização de serviços de cuidados para } \\
\text { mulheres com essas condições }\end{array}$ & $\begin{array}{l}\text { Descritivo- } \\
\text { quantitativo }\end{array}$ & N-6 \\
\hline Mandl et al. & Descrever a qualidade do atendimento à incontinência em lares de idosos & Quantitativo & $\mathrm{N}-6$ \\
\hline Glazener et al. & $\begin{array}{l}\text { Determinar os efeitos em longo prazo (12 anos) de uma intervenção } \\
\text { conservadora conduzida por enfermeiros para a IU pós-natal }\end{array}$ & $\begin{array}{l}\text { Ensaio clínico } \\
\text { controlado } \\
\text { randomizado }\end{array}$ & $\mathrm{N}-2$ \\
\hline Teunissen et al. & $\begin{array}{l}\text { Determinar a eficácia de introduzir uma enfermeira no cuidado da IU e } \\
\quad \text { explorar as razões das mulheres por não completar o tratamento }\end{array}$ & $\begin{array}{l}\text { Ensaio clínico sem } \\
\text { randomização }\end{array}$ & N-3 \\
\hline Gagne et al. & $\begin{array}{l}\text { Desenvolver, implementar e avaliar a IU em um programa de autogestão } \\
\text { para mulheres residentes na comunidade de } 55 \text { anos ou mais com IU na } \\
\text { Coréia do Sul rural. }\end{array}$ & $\begin{array}{l}\text { Ensaio clínico sem } \\
\text { randomização }\end{array}$ & $N-3$ \\
\hline Choi et al. & $\begin{array}{c}\text { Avaliar se o cuidado de continência realizado por enfermeiras é eficaz na } \\
\text { melhoria dos resultados para pacientes adultos chineses com sintomas } \\
\text { do trato urinário inferior }\end{array}$ & $\begin{array}{l}\text { Ensaio clínico } \\
\text { randomizado }\end{array}$ & $\mathrm{N}-2$ \\
\hline
\end{tabular}

Adaptado de Mendes et al. (2008).

Pesquisa realizada na Holanda tinha como objetivo avaliar a gravidade da IU sobre a qualidade de vida (QV) das pessoas encaminhadas pelos clínicos e atendidas por enfermeiros treinados. Essa pesquisa foi realizada por meio de um instrumento utilizado para avaliar os pacientes e, concomitantemente, monitorar sua resposta às intervenções ao longo do tempo. Em seguida, aplicou-se um questionário que visava a conhecer os impactos que a IU ocasionava em suas vidas. O estudo foi realizado em dois momentos; no primeiro, os pesquisadores avaliaram as queixas e os hábitos de vidas dos participantes e foram realizadas algumas intervenções; no segundo, após 3 meses, fez-se uma reavaliação a fim de se verificar os resultados das intervenções ${ }^{12}$.

Ao final do estudo, os autores constataram que houve melhora significativa acerca das queixas dos pacientes no que tange às dimensões sociais, emocionais e ao bem-estar, enquanto, com relação às viagens e à mobilidade, não foi observado nenhum resultado significativo. Além disso, percebeu-se a existência de correlação entre os alimentos ingeridos pelos participantes e a IU, sendo que a ingestão de 3,6 xícaras por dia de cafeína e o consumo excessivo de álcool demonstraram ser fatores importantes que podem afetar negativamente a IU. No que concerne à atuação dos enfermeiros, os resultados desse estudo apontaram que, quando esses profissionais são devidamente capacitados, podem ser capazes de influenciar na melhora da QV e aumentar a motivação das pessoas com IU, contribuindo, assim, para um tratamento mais eficaz ${ }^{12}$.

Os exercícios musculares, por meio de contração e relaxamento da musculatura do assoalho pélvico visando a melhora da rigidez, da força, da resistência, da coordenação e da função dos músculos; a modificação da dieta por meio da restrição hídrica noturna e da redução do consumo de álcool e de líquidos com cafeína; e o treinamento da bexiga, no 
qual não se podiam utilizar o banheiro por um determinado período de tempo, de forma que o intervalo entre uma micção e outra fosse aumentado, são intervenções realizadas por enfermeiros que tiveram melhoria nos desfechos clínicos das pessoas com IU e melhora na qualidade de vida ${ }^{13}$.

As fraldas superabsorventes, os sprays utilizados para formação de película de polímero e os lenços umedecidos e impregnados com dimeticona a 3\% constituíram estratégias eficientes na prevenção e no tratamento da dermatite associada à $\mathrm{IU}^{14}$.

A água e o sabão tiveram desempenho ruim na prevenção e no tratamento da dermatite associada à IU. A Calmoseptina com $20 \%$ de óxido de zinco em sua composição apontou melhora no tratamento da dermatite. O Cavilon e outro protetor de barreira estavam sendo testados em alguns estudos e por isso ainda não se sabe se são ou não eficazes ${ }^{15}$.

Quanto aos tipos de sondas e as melhores técnicas de cateterismo para prevenção das complicações da IU, houve escassez de estudos que revelassem que a incidência de infecção do trato urinário possa melhorar com algum tipo de técnica, estratégia ou sonda. Destacou-se a importância do plano de cuidados individualizados para identificar a frequência apropriada de cateterismo e que a sondagem vesical intermitente oferece mais vantagens que a sondagem permanente, pela maior oportunidade de autocuidado e independência de indivíduos, redução do risco de complicações comuns associadas à sonda permanente, redução da necessidade de instrumentos e artefatos, como bolsa de drenagem, maior liberdade de expressão para a sexualidade e possibilidade de reduzir os sintomas urinários (frequência, urgência, incontinência) entre cada intervalo do cateterismo ${ }^{16}$.

Na prática clínica, utiliza-se o pessário vaginal, que se trata de uma esponja de poliuretano confeccionado em três tamanhos, colocada na vagina com auxílio de um aplicador. Os enfermeiros ou fisioterapeutas alegaram não terem recebido treinamento para manusear os pessários, enquanto os médicos foram significativamente mais propensos a relatar os cuidados com pessários ${ }^{17}$.

\section{Terceira dimensão do cuidado: gerencial}

Apenas um artigo foi encontrado nessa temática, sendo que esse foi categorizado também como assistencial. $\mathrm{O}$ estudo realizado em 16 lares de idosos descreveu a qualidade do atendimento à IU em lares de idosos. Avaliaram-se diversos indicadores estruturais e humanos que interferiam na assistência e na funcionalidade das instituições.

No que tange à disponibilidade de indicadores estruturais em nível institucional, observou-se que as diretrizes para tratamento e o item especialista, utilizado para atualizar as diretrizes, estavam frequentes em 10 instituições (62\%). No indicador "protocolos para gerenciamento de produtos de incontinência”, 15 (93\%) dos lares de idosos adotaram essa política no padrão de qualidade. Os folhetos informativos que abordavam a temática IU estavam presentes em apenas três instituições $(18,75 \%)$ e, acerca da disponibilidade dos indicadores estruturais no nível setor/alas, evidenciou-se que apenas seis $(36 \%)$ tinham um profissional de enfermagem para as pessoas idosas acometidas por IU. Com relação às intervenções de enfermagem, o resultado mostrou que $85 \%$ das pessoas com IU utilizavam almofadas descartáveis e outros $51 \%$ adotavam hábitos comportamentais para diminuir suas perdas urinárias. Outros indicadores, como avaliação de medicamentos, trocas de fraldas e treinamento da musculatura pélvica apareceram como intervenções poucas vezes utilizadas ${ }^{18}$.

Assim, constatou-se que os resultados deste estudo fornecem uma visão inicial da qualidade do cuidado da incontinência em relação à estrutura, nível de processo e indicadores de resultados em asilo austríaco e concluíram que os indicadores estruturais estão limitados em lares de idosos austríacos. A prevalência de todos os tipos de incontinência ainda era alta, o que demanda a necessidade de melhorias no cuidado da incontinência em asilo austríaco, a exemplo de curso de atualização sobre a temática ${ }^{18}$.

\section{DISCUSSÃO}

Quanto à dimensão educacional, essa deve ser entendida como uma prática com finalidade de proporcionar a melhoria das condições de vida e de saúde por meio de orientações às pessoas e familiares ${ }^{19}$.

Posto isso, as pessoas precisam adquirir autonomia para identificar e utilizar as formas e os meios para preservar e melhorar sua vida. Esse processo deve envolver a negociação entre perspectiva dos profissionais de saúde ajustada às necessidades e prioridades definidas pelo próprio paciente ${ }^{20}$. 
Transpondo esse conceito para o cuidado às pessoas com IU, é fundamental a consulta de enfermagem para conhecer as necessidades, a fim de propor ações de educação que possam prevenir, tratar e melhorar suas queixas, de modo que haja mudanças comportamentais em direção à melhoria da qualidade de vida. Para tanto, faz-se necessário que o enfermeiro acompanhe a evolução científica e tecnológica, adote estratégias e habilidades para transformar e meios para facilitar as ações educativas.

Alguns enfermeiros entrevistados demonstraram a importância de orientar os cuidados relacionados à alimentação saudável, uso correto de medicamentos, controle das doenças crônicas, medidas para prevenir constipação, medidas para evitar o tabagismo e álcool, entre outros. Na concepção dos enfermeiros, esses fatores de risco poderiam prevenir e/ou minimizar os sintomas. Acredita-se que há necessidade de investir mais na capacitação dos profissionais para que a saúde do idoso seja abordada de forma integral, promovendo melhora no bem-estar e qualidade de vida9.

Percebeu-se que as atividades de educação desenvolvidas pelos enfermeiros em relação à IU são pautadas em ações de promoção e prevenção de doenças e que há falta de ações de prevenção e promoção em saúde. E, ainda, dos 13 enfermeiros envolvidos no cuidado, apenas um trouxe a importância de promover o autocuidado 8 .

Para o desenvolvimento das ações de educação para trabalhar o tema IU, utilizou-se da musicoterapia, com canções populares que abordavam a prevenção em saúde, tornando as sessões de educação divertidas e eficazes, saindo da rotina de papéis e lápis ${ }^{10}$.

A dimensão assistencial caracteriza-se pela implementação de ações de cuidado de enfermagem, tendo como objeto de intervenção as necessidades de cuidado, e sua finalidade é prestar cuidado numa perspectiva da integralidade.

Ressalta-se que o enfermeiro tem que se apropriar de quatro componentes para prestar assistência especializada na abordagem da IU: teórico filosófico, teórico conceitual (fisiopatologias das incontinências); alicerces para o cuidar taxonômicos [American Nursing Diagonosis Ssociation (Nanda), Nursing Interventions Classification (NIC) e Nursing Outcomes Classification (NOC)] e legais [Sistematização da Assistência de Enfermagem ( $S A E$ ) e processo de enfermagem]. Nesse entendimento, os fatores relacionados e as características definidoras viabilizam a investigação e determinação de condutas do enfermeiro na assistência à pessoa com IU ou com risco de desenvolvê-la ${ }^{21}$.
Foram utilizados, como intervenção de enfermagem, os exercícios da musculatura pélvica e o treinamento da bexiga. Embora os resultados não tenham sido satisfatórios, atribuíram o insucesso à falta da longitudinalidade no acompanhamento dos participantes ${ }^{11}$.

Em contrapartida, um estudo constatou resultados positivos com as intervenções realizadas pelos enfermeiros por meio de exercícios e treinamento e as modificações dos hábitos de vida. Atribuíram também, aos resultados satisfatórios, à relação que se estabeleceu entre os participantes, principalmente entre as mulheres e os profissionais, tendo em vista que os participantes encontraram espaço para manifestar suas queixas relacionadas à $\mathrm{IU}^{13}$.

Entretanto, resultados similares foram encontrados pelo fato de as intervenções por meio de exercícios e treinamentos terem sido eficazes e quão importantes são os enfermeiros nesse processo ${ }^{12,13}$.

Os resultados dos estudos de intervenção revelaram que os exercícios e treinamentos para a IU são eficazes, assim como as ações desenvolvidas pelos enfermeiros e a importância do acolhimento, para que as pessoas possam encontrar espaços para a dialogicidade e para manifestar suas necessidades. Cabe ressaltar que os três estudos são internacionais e apontam importante lacuna do conhecimento no âmbito nacional.

No Brasil, há mais de 10 anos, na Faculdade de Enfermagem da Unicamp, tem sido realizado o Programa de Reabilitação do Assoalho Pélvico (PRAP), com a finalidade de treinar profissionais enfermeiros e graduandos de enfermagem para atuar com essa prática, fornecendo, assim, assistência de qualidade e resolutiva. Atualmente, há outros profissionais, fisioterapeutas e educadores físicos, envolvidos no projeto ${ }^{22}$.

Estudos constataram que as fraldas superabsorventes, os lenços com dimeticona a $5 \%$, o spray com película de polímero e a Calmoseptina 20\% com óxido de zinco demonstraram boa eficácia no tratamento das dermatites associadas à IU ${ }^{14,15}$. Em contrapartida, a água com sabão não é adequada para uso em dermatite e, embora o Cavilon tenha sido utilizado no tratamento da dermatite, os resultados do estudo com utilização desse produto ainda não tinham sido concluídos ${ }^{15}$.

Porém, há que ressaltar que, em ambas as revisões, os estudos selecionados foram internacionais e de baixa evidência, o que demonstra a necessidade de estudos desenvolvidos por enfermeiros e com nível alto de evidência para testar os melhores produtos para prevenção e tratamento da dermatite associada à IU e sustentar a prática clínica. 
Ainda em relação ao uso de tecnologias, dispositivos e produtos, um estudo foi inconclusivo em relação aos tipos de sondas e técnicas para a prevenção da IU. O autor concluiu as vantagens em relação ao incentivo ao autocuidado e redução do risco de infecção da sondagem intermitente em relação à sondagem permanente ${ }^{16}$.

Um estudo revela que, apesar de os pessários serem utilizados e recomendados para o tratamento da IU, o enfermeiro ainda não se sente capacitado para utilização desse dispositivo, enquanto os médicos têm demonstrado maior segurança, seguidos pelos fisioterapeutas ${ }^{17}$.

Os resultados dos estudos revelaram importante lacuna do conhecimento e a necessidade de desenvolvimento de pesquisas clínicas sobre o manejo da IU realizado pelos enfermeiros sobre a avaliação da eficácia de produtos e as melhores técnicas para minimizar sua ocorrência, principalmente relacionada aos materiais e à técnica da sonda vesical, tendo em vista que é uma técnica privativa do enfermeiro.

No que concerne à dimensão gerencial, evidenciou-se a escassez de estudos, embora a gerência constitua importante dimensão que se articula às demais dimensões do cuidado.

O estudo encontrado na dimensão gerencial constatou, como importantes indicadores da qualidade do cuidado, a disponibilidade e a manutenção das almofadas e dos protetores de cadeiras e camas, dos folhetos informativos que permitiram às pessoas institucionalizadas compreender seus sinais, sintomas, causas e maneiras de tratar, os horários para visitas ao banheiro, disponibilidade de enfermeira e de protocolos para utilização de produtos para incontinência e os registros de enfermagem ${ }^{18}$.

Os enfermeiros, para melhor gerenciar suas atividades diárias, têm adotado, como importantes ferramentas, os protocolos e os Procedimentos Operacionais Padrão (POPs). Esse instrumento, elaborado a partir de conhecimento técnico, científico e legal, permite a padronização da assistência, uma vez que normatiza, fundamenta, padroniza, organiza e qualifica qualquer atividade prestada para que haja eficiência e eficácia. Também é importante considerar que a educação continuada é vista como uma estratégia que permite o desenvolvimento das pessoas para que os objetivos da instituição sejam alcançados.

Dos 11 artigos selecionados para esta revisão, apenas um preocupou-se em avaliar os indicadores relacionados à assistência dos profissionais às pessoas com IU. Percebe-se, também, a existência da dicotomia entre gerência e assistência, o que torna contraproducente as dimensões do cuidado ${ }^{13}$.
Embora a IU seja considerada importante questão de saúde pública, no Brasil, são escassas as pesquisas desenvolvidas na temática, principalmente pelo enfermeiro. Há, portanto, importante lacuna a ser preenchida com estudos com maior rigor metodológico e altos níveis de evidência que possibilitem sustentar a prática clínica do enfermeiro, com vistas à promoção, à prevenção, ao tratamento e à reabilitação da IU.

\section{CONCLUSÃO}

Conclui-se que o conhecimento do enfermeiro na IU é incipiente e que esse tende a desenvolver suas ações na dimensão assistencial com predomínio no tratamento e recuperação. Essa realidade aponta para importante lacuna. Espera-se que o enfermeiro atue mais e assertivamente na educação em saúde, com ações de prevenção. Assim, os gastos financeiros, o tempo de trabalho e o desgaste emocional seriam minimizados.

No que tange às limitações deste estudo, observou-se que a maioria dos artigos analisados era de metodologia descritiva, sendo, portanto, classificada com baixo nível de evidência. Além disso, verificou-se a escassez de publicações no que se refere aos dispositivos oferecidos no mercado para atender o público com a condição de IU, seja na prevenção, na reabilitação ou na melhora da qualidade de vida, o que impossibilita aos enfermeiros a padronização fundamentada em níveis de evidência sobre os melhores produtos e técnicas para o manejo da IU.

Dada a escassez de estudos e o conhecimento incipiente do enfermeiro sobre essa importante condição, sugere-se a realização de estudos com fortes níveis de evidência, a inclusão da temática nos cursos de graduação e o desenvolvimento de programas no âmbito da atenção primária de saúde.

\section{CONTRIBUIÇÃO DOS AUTORES}

Conceitualização, Fava SMCL; dos Reis SC; Oliveira TS; Dias JF e Sanches RS; Metodologia, Fava SMCL; dos Reis SC; Oliveira TS; Dias JF; Sanches RS e Dazio EMR; Investigação, Fava SMCL; dos Reis SC; Oliveira TS e Dias JF; Redação - Primeira versão, Fava SMCL; dos Reis SC; Oliveira TS; Dias JF; Sanches RS e Dazio EMR; Redação - Revisão \& Edição, Fava SMCL; Sanches RS e Dazio EMR; Recursos, Fava SMCL; Supervisão, Fava SMCL.. 


\section{REFERÊNCIAS}

1. International Continence Society. Recommendations of the International Scientific Committee: evaluation and treatment of urinary incontinence, pelvic organ prolapse and faecal incontinence. $4^{\text {a }}$ International Consultation on Incontinence; 2009. [citado 10 jan 2018]. Disponível em: http://www.ics. org/Publications/ICl 4/files-book/recommendation.pdf

2. Bolina AF, Dias FA, Santos NMF, Tavares DMS. Incontinência urinária autorreferida em idosos e seus fatores associados. Rev RENE. 2013;14(2):49-55.

3. Santos KFO. Qualidade de vida de pessoas idosas com incontinência urinária [dissertação]. João Pessoa (PB): Universidade Federal da Paraíba, Centro de Ciências da Saúde; 2013.

4. Mendes KDS, Silveira RCCP, Galvão CM. Revisão integrativa: método de pesquisa para a incorporação de evidências na saúde e na enfermagem. Texto Contexto Enferm. 2008;17(4):758-64.

5. Ursi ES. Prevenção de lesões de pele no perioperatório: revisão integrativa da literatura. Rev Latino-am Enfermagem. 2005;14(1):124-31.

6. Melnyk BM, Fineout OF. Making the case for evidence-based practice. Evidence-based practice in nursing e healthcare. A guide to best practice. In: Melnyk BM, Fineout-Overholt E. Evidence-based practice in nursing \& health care. A guide to best practice. Philadelphia: Lippincott Willians \& Wilkins; 2005. p. 3-24.

7. Hausmann M, Peduzzi M. Articulação entre as dimensões gerencial e assistencial do processo do trabalho do enfermeiro. Texto Contexto Enferm. 2009;18(2):258-65.

8. BittencourtJR, Lopes IR, Júnior JJS, Oliveira NRR, Teles MAB, Luna PMR. Assistência de enfermagem a idosos com incontinência Urinária. Rev enferm UFPE. 2017;11(2):966-72. https://doi. org/10.5205/reuol.10263-91568-1-RV.1102sup201712

9. Tomasi AVR, Santos SMA, Honório GJS, Locks MOH. Incontinência urinária em idosas: práticas assistenciais e proposta de cuidado âmbito da atenção primária de saúde. Texto contexto - enferm. 2017;26(2):e6800015. https://doi. org/10.1590/0104-07072017006800015

10. Gagne JC, So A, Wu B, Palmer MH, Connell ESMC. The effect of a urinary incontinence self-management program for older women in South Korea: a pilot study. Int J Nurs Sci. 2015;2(1):39-46. https://doi.org/10.1016/j.ijnss.2015.01.002

11. Glazener CMA, MacArthur C, Hagen S, Elders A, Lancashire $\mathrm{R}$, Herbison GP et al. Twelve-year follow-up of conservative management of postnatal urinary and faecal incontinence and prolapse outcomes: randomised controlled trial. BJOG. 2013;121(1):112-20.
12. Teunissen DTAM, Stegeman MM, Bor HH, Langro-Janssen TALM. Treatment by a nurse practitioner in primary care improves the severity and impact of urinary incontinence in women. BMC Urol. 2015;15:51. https://doi.org/10.1186/ s12894-015-0047-0

13. Choi EPH, Chin WY, Lam CLK, Wan EYF, Chan AC, Chan KHY. Evaluation of the effectiveness of nurse-led continence care treatments for Chinese primary care patients with lower urinary tract symptoms. Plos One. 2015;10(6);e0129875. https://doi.org/10.1371/journal.pone.0129875

14. Rosa LHT, Souza CM, Lima CHL, Boggiod ESB, Santose FC, Carbonif $C$ et al. Prevalência da incontinência urinária em idosos de Porto Alegre-RS. Rev Geriatr Gerontol. 2014;9(2):112-7.

15. Beeckman D, Damme NV, Schoonhoven L, Lancker AV, Kottner J, Beele $\mathrm{H}$. et al. Interventions for preventing and treating incontinence-associated dermatitis in adults. Cochrane Database Syst Rev. 2016;11. https://doi. org/10.1002/14651858.CD011627

16. Torres A, Angustias M. Medidas preventivas para evitar complicaciones de salud derivadas del uso de sondajes vesicales en pacientes lesionados medulares. Enferm glob. 2013;12(30): 370-78.

17. Bugge C, Hagen S, Thakar R. Vaginal pessaries for pelvic organ prolapse and urinary incontinence: a multiprofessional survey of practice. Int Urogynecol J. 2013;24(6):1017-24. https://doi.org/10.1007/s00192-012-1985-7

18. Mandl M, Halfens RJ, Lohrmann C. Incontinence care in nursing homes: a cross-sectional study. J Adv Nurs. 2015;71(9):2142-52. https://doi.org/10.1111/jan.12676

19. Pressoto GV, Ferreira MBG, Contim D, Simões ALA. Dimensões do trabalho do enfermeiro no contexto hospitalar. Rev RENE. 2011;15(5):760-70. https://doi. org/10.15253/2175-6783.2014000500005

20. Lopes APF. Cuidado e empoderamento: a construção do sujeito responsável por sua saúde na experiência do diabetes. Saude soc. 2015;24(2):486-500. https://doi. org/10.1590/S0104-12902015000200008

21. Martins NA. Pessoas idosas e incontinência urinária: trajetória da proposição de um modelo de sistematização da assistência especializada em enfermagem [dissertação]. Juiz de Fora (MG): Universidade Federal de Juiz de Fora, Programa de Pós-Graduação em Enfermagem; 2014.

22. Lopes MHBM, Costa JN, Lima JLDA, Oliveira LDR, Caetano AS. Programa de reabilitação do assoalho pélvico: relato de dez anos de experiência. Rev Bras Enferm. 2017;70(1):219-23. https://doi.org/10.1590/0034-7167-2016-0257 